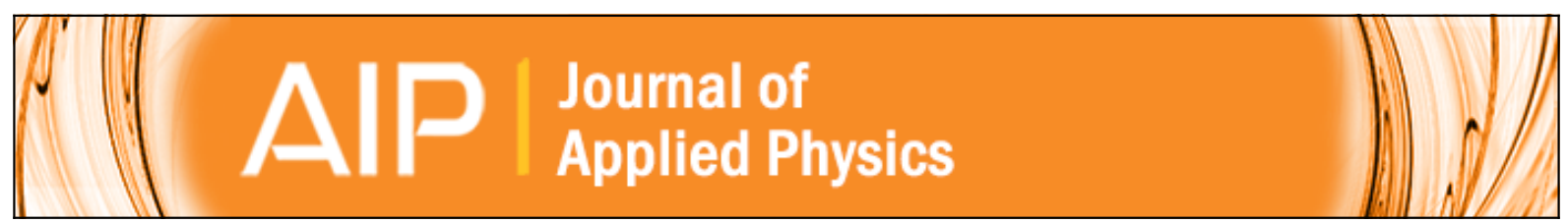

On the temperature dependence of the magnetoresistance of ferromagnetic alloys

L. Berger, P. P. Freitas, J. D. Warner, and J. E. Schmidt

Citation: Journal of Applied Physics 64, 5459 (1988); doi: 10.1063/1.342347

View online: http://dx.doi.org/10.1063/1.342347

View Table of Contents: http://scitation.aip.org/content/aip/journal/jap/64/10?ver=pdfcov

Published by the AIP Publishing

$\underset{\text { Aublosing }}{A}$ Re-register for Table of Content Alerts

Create a profile. 


\title{
On the temperature dependence of the magnetoresistance of ferromagnetic alloys
}

\author{
L. Berger, P. P. Freitas, J. D. Warner, and J. E. Schmidt \\ Physics Department, Carnegie-Melion University, Pittsburgh, Pennsyluania 15213
}

\begin{abstract}
The ferromagnetic anisotropy of resistivity $\Delta \rho / \rho_{0}=\left(\rho_{1}-\rho_{1}\right) / \rho_{0}$ depends strongly on the nature of the dominant electron scattering centers, and is usually larger for impurity scatiering than for phonon scattering. This explains why $\Delta \rho / \rho_{0}$ decreases with increasing temperature $T$ in crystalline transition-metal alloys. Parker has proposed an expression for $\Delta \rho / \rho_{0}$ as a function of $T$, based on Matthiessen's rule. It contains the parameters $\left(\Delta \rho / \rho_{0}\right)_{\mathrm{im}}$ and $\left(\Delta p / \rho_{0}\right)_{p h}$, representing the $\Delta p / p_{0}$ values for impurity scattering and phonon scattering, respectively. In an alloy series with weak electron scattering, such as $\mathrm{Fe}-\mathrm{Co},\left(\Delta \rho / \rho_{0}\right)_{\mathrm{ph}}$ is found experimentally to be positive and equal to a fixed fraction $=\frac{8}{4}$ of $\left(\Delta \rho / \rho_{0}\right)$ im. On the other hand, in crystalline alloy series with strong, resonant electron scattering, such as $\mathrm{Ni}-\mathrm{Fe}$, Fe-Cr, Fe-V, $\left(\Delta \rho / \rho_{0}\right)_{p h}$ is found to be negative and has a minimum at the composition where $\left(\Delta \rho / \rho_{0}\right)_{\mathrm{im}}$ is maximum.
\end{abstract}

\section{IRTRODUCTIOK}

The ferronagnetic anisotropy of resistivity is defined as $\Delta \rho / \rho_{0}=\left(\rho_{i \mid}-\rho_{1}\right) /\left(\rho_{l i} / 3+2 \rho_{1} / 3\right)$, where $\rho_{i j}$ and $\rho_{j}$ are the resistivity values when the magnetization is parallel and perpendicular to the current, respectively. It arises from the combination of spin-orbit interaction and $s-d$ electron scattering. In most crystalline transition-metal alloys, $\Delta \rho_{0} / \rho_{0}$ is positive, and a decreasing function of the temperature $T$. The present paper concerns such crystalline alloys, but not amorphous materials.

Fert, Campbell, and Jaoul proposed ${ }^{\prime}$ the following relation in the case of nickel alloys at $T \simeq 0$ :

$$
\Delta \rho / \rho_{0}=\gamma\left(\rho_{1} / \rho_{1}-\hat{1}\right)
$$

where $\rho_{1}$ and $\rho_{1}$ are the resistivities for the spin-down and spin-up bands, respectively, and $\gamma \approx 0.0 \mathrm{l}$. The condition $\rho_{1} / \rho_{1} \geqslant 1$ is assumed. A similar relation, where $\rho_{1} / \rho_{1}$ is replaced by $\rho_{\uparrow} / \rho_{\curlywedge}$, holds for Fe-based alloys in the limit $\rho_{1} / \rho_{1}>1$.

At $T>0$, these authors assumed' a "spin-mixing" resistivity $\rho_{1},(7)$ to exist, which couples the spin-up and spindown currents so that Eq. (1) has to be modified. The introduction of $\rho_{3}$ is equivalent to having $\rho_{1} / \rho_{1}$ approach the value of 1 , leading to a reduction of $\Delta \rho / \rho_{0}$. The gradual increase of $\rho_{t,}$ with increasing $T$ explains why $\Delta \rho / \rho_{0}$ decreases at increasing temperatures. It was also suggested that $\rho_{i}$, arises from electron-magnon or electron-electron scattering. Unfortunately, these two scattering mechanisms are usually very weak at $300-400 \mathrm{~K}$, as compared to electron-phonon scattering, and not likely to be significant.

Both electron-magnon and electron-electron scattering give rise to very inelastic collisions. As a resuit, the Lorenz number $L(T)=\kappa_{0} \rho_{0} / T$, where $\kappa_{0}$ is the zero-field thermal conductivity and $\rho_{0}$ the zero-field resistivity, should be ${ }^{2}$ considerably smaller than the value $L_{\mathrm{el}}=2.44 \times 10^{-8} \mathrm{~W}^{2} \mathrm{~K}^{-2}$ for elastic collisions, if either of these two mechanisms were dominant at $300-400 \mathrm{~K}$. However, existing data ${ }^{3}$ for $\mathrm{Fe}$, $\mathrm{Ni}$, and $C o$ show that $L(T)$ rises, and approaches $L_{\mathrm{el}}$ above the Debye characteristic temperature $\approx 400 \mathrm{~K}$, as expected if electron-phonon scattering dominates. This confirms our conclusion that no significant spin-mixing resistivity $\rho_{i 1}(T)$ exists in nickel, iron, or cobalt at $300-400 \mathrm{~K}$. This conclusion should hold a fortior in alloys whete additional scattering by impurities is present.

Insiead, we attribute the temperature dependence of $\Delta p / \rho_{i}$ to the well-known fact that $\Delta \rho / \rho_{0}$ depends strongly on the nature of the dominant electron scattering centers. The characteristic value of $\Delta \rho / \rho_{0}$ for phonon scattering will be called $\left(\Delta \rho / \rho_{0}\right)_{\mathrm{ph}}$, and is usually smaller than the characreristic value $\left(\Delta \rho / \rho_{0}\right)_{\text {ir }}$ for impurity scattering.

Matthiessen's rule is valid whenever conduction by one specific group of electrons dominates over that of other groups, i.e., when $\rho_{1} / \rho_{1} \gg 1$ or $\rho_{1} / \rho_{2} \gg 1$. It states that the resistivity $\rho_{\mathrm{im}}$ caused by impurity scattering and the resistivity $\rho_{p h}(T)$ caused by electron-phonon scattering are additive, so that $\rho_{0}(T)=\rho_{\mathrm{in}}+\rho_{\mathrm{ph}}(T)$. An expression for $\Delta \rho /$ $\rho_{0}$ at arbitrary temperature $T$, based on Mathiessen's rule, has been derived by parker ${ }^{4}$ and reformulated by us':

$\frac{\Delta \rho}{\rho_{0}}(T)=\left(\frac{\Delta \rho}{\rho_{0}}\right)_{p h}+\left[\left(\frac{\Delta \rho}{\rho_{0}}\right)_{\mathrm{im}}-\left(\frac{\Delta \rho}{\rho_{0}}\right)_{p=1}\right] \frac{\rho_{0}(4.2)}{\rho_{0}(T)}$.

where $\rho_{0}(4.2) \approx \rho_{\mathrm{im}}$. Parker's formula also has a factor containing the saturation magnetization, but this is almost constant if $T$ remains suficiently far below the Curie point.

\section{PARKER PLOTS FOR FE-COALLOYS}

We have measured $\Delta \rho / \rho_{0}$ and $\rho_{0}$ on an annealed and furnace-cooled $\mathrm{Fe}_{18} \mathrm{Co}_{82}$ sample 6 between 79 and $295 \mathrm{~K}$, in a variable-temperature cryostat. $A 1 s 0_{3} \Delta \rho / \rho_{0}$ and $\rho_{0}$ have been measured in liquid baths at 4.2 and $77 \mathrm{~K}$, and in air at $295 \mathrm{~K}$. We plot the measured $\Delta \rho / \rho_{0}$ vs $\rho_{0}(4.2) / \rho_{0}(T)$ in Fig. $1(a)$. This kind of graph will be called a "Parker plot." If Parker's law [Eq. (2)] is valid, data points should be located on a straight line. We see that this is the case within the accuracy of the data, at $77 \mathrm{~K}$ and above.

On the other hand, the data point for $4.2 \mathrm{~K}$ is located below the straight line in Fig. 1(a). Similar deviations from 

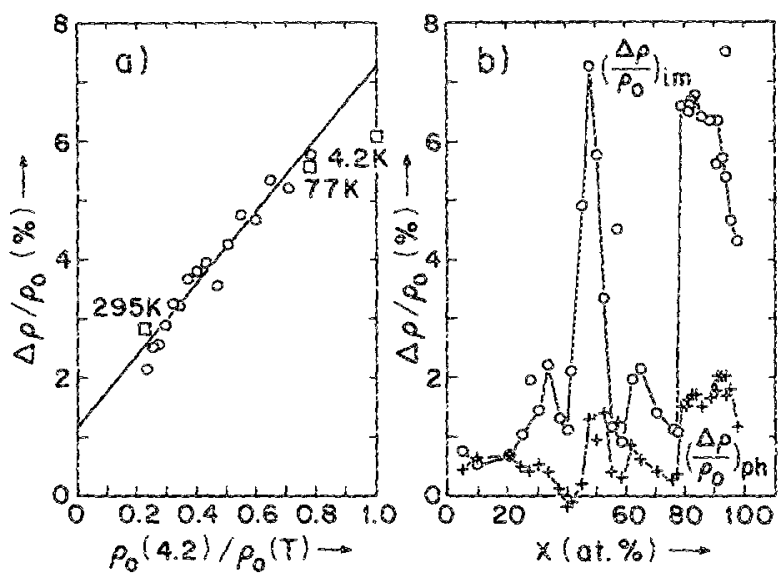

FIG. 1. (a) Parker plot for a $F \varepsilon_{18} \mathrm{Co}_{6}$, alloy. Circles represent $\Delta \rho / \rho_{0}$ values obtaned in a variabie-temperature cryostat, and squares are values obtained in liquid baths or air. (b) Circies represent $\left(\Delta \rho / p_{c}\right)_{m}$ values for $\mathrm{Fe}_{100}$. $\mathrm{CO}_{\lambda}$ as a function of $x$, while crosses represent $\left(\Delta \rho / \rho_{0}\right\}_{p h}$.

Parker's law are observed at low temperatures in many alloy series, especially those with low-resistivity $\rho_{0}$ and high saturation magnetization $M_{S}$. They are caused by the internal field $B=M_{S}$ which creates a Lorentz force curving the electron trajectories, in turn leading to a negative contribution to $\Delta \rho / \rho_{0}$ if the latter is measured at ferromagnetic saturation.

The intercept of the straight line on the left-hand vertical axis gives $\left(\Delta \rho / \rho_{0}\right)_{\mathrm{ph}}$, here equal to $+1.2 \%$. Similariy, the intercept on the right-hand vertical axis gives $\left(\Delta \rho / \rho_{0}\right)_{\mathrm{im}}=+7.25 \%$

The values of $\Delta \rho / \rho_{0}$ and $\rho_{0}$ have also been measured ${ }^{6,8}$ in a series of $\mathrm{Fe}_{100 \ldots x} \mathrm{Co}_{x}$ alloys at $4.2,77$, and $295 \mathrm{~K}$. Parker plots similar to Fig. 1(a) have been drawn for all 37 samples. By drawing straight lines through the two data points at 77 and $295 \mathrm{~K},\left(\Delta \rho / \rho_{0}\right)_{\mathrm{ph}}$ and $\left(\Delta \rho / \rho_{0}\right)_{\mathrm{im}}$ have been deter. mined for all samples as above, and are dispiayed as a function of cobalt concentration $x$ in Fig. $1(b)$. Some data points are too close together to be seen in Fig. 1(b). For the reason described above, the data point for $4.2 \mathrm{~K}$ often falls below the straight line. We see in Fig. $1(b)$ that $\left(\Delta \rho / \rho_{0}\right)_{\text {in }}$ and $\left(\Delta \rho / \rho_{0}\right)_{\text {ph }}$ are usually positive. Aiso, samples with large $\left(\Delta \rho / \rho_{0}\right)_{\text {in }}$ tend to have large $\left(\Delta \rho / \rho_{0}\right)_{\mathrm{ph}}$. Except for Fe-rich samples, $\left(\Delta \rho / \rho_{0}\right)_{p h}$ is roughly a fixed fraction $\simeq \frac{1}{4}$ of $\left(\Delta \rho / \rho_{0}\right)_{\text {im }}$. The Smit theory of $\Delta \rho / \rho_{0}$, based on the first Born approximation, predicts $\rho_{1} / \rho_{,} \propto D_{1} / D_{1}$, where $D_{1}$ and $D$, are the $3 d$ densities of states for spin down and spin up. Using Eq. (1) in the limit $\rho_{1} / \rho_{i} \gg 1$, this implies $\Delta \rho /$ $\rho_{0} \propto D_{1} / D_{1}$. That result should apply to both $\left(\Delta \rho / \rho_{0}\right)_{\text {im }}$ and $\left(\Delta \rho / \rho_{0}\right)_{p h}$, though the proportionality coefficients will be different. In other words, $\left(\Delta \rho / \rho_{0}\right)_{\mathrm{m}}$ and $\left(\Delta \rho / \rho_{0}\right)_{\mathrm{ph}}$ are predicted to vary in similar fashion through the Fe-Co series, reflecting variations of $D$ and $D$. with composition. This agrees with the data of Fig. I(b). For example, the sharp peaks of $\left(\Delta \rho / \rho_{0}\right)_{\mathrm{im}}$ and $\left(\Delta \rho / \rho_{0}\right)_{p t}$ in Fig. 1(b) correspond ${ }^{6,8}$ to minima of $D$, caused by atomic ordering.

\section{PARKER PLOTS FOR NIFE, FECT, AND FEV ALLOYS}

Measurements of $\Delta \rho / \rho_{0}$ have been done on $\mathrm{Ni}_{200, x} \mathrm{Fe}_{x}$ alloys at $\approx 290$ and $4.2 \mathrm{~K}$ (or $20 \mathrm{~K}$ ) by several authors, ${ }^{1,0}$ similarly for $p_{0}$ measurements. "Unfortunately, data at 77 $K$ are not available. We have drawn smooth curves representing average values of $\Delta \rho / \rho_{0}$ and $\rho_{0}$ as a function of $x$. parker plots have been constructed for $x=5,10,15, \ldots, 50$ at. $\%$ Fe. Values of $\left(\Delta p / \rho_{0}\right)_{\mathrm{ph}}$ and $\left(\Delta \rho / \rho_{0}\right)_{\mathrm{im}}$ have been determined for all such compositions by drawing straight lines through the 4.2 and $290 \mathrm{~K}$ points, and are shown in Fig. $2(a)$. In addition, we show the $\Delta \rho / \rho_{0}$ values measured on pure nickel ${ }^{12}$ at $\approx 290 \mathrm{~K}$. These should be identified with $\left(\Delta \rho / \rho_{0}\right)_{p h}$ since impurity scattering is absent.

We see that $\left(\Delta \rho / \rho_{0}\right)_{\mathrm{im}}$ is positive at all compositions, and has a maximum at $x \approx 15 \% \mathrm{Fe}$. On the other hand, $\left(\Delta \rho / \rho_{0}\right)_{\mathrm{ph}}$ becomes negative as the iron concentration increases, and has a minimum at a value of $x$ somewhat larger than the one where $\left(\Delta \rho / \rho_{0}\right)_{\text {im }}$ is maximum. This behavior of $\left(\Delta p / p_{0}\right)_{\mathrm{ph}}$ is very different from the one observed in $\mathrm{Fe}-\mathrm{Co}$ [Fig. $1($ b) ], and camnot be explained merely in terms of $D$. and $D_{1}$ variations.

We have measured $\Delta \rho / \rho_{0}$ and $\rho_{0}$ at 299,77 , and $4.2 \mathrm{~K}$ on $\mathrm{Fe}_{100)-x} \mathrm{Cr}_{x}$ alloys with $x=2.3,5.1,12.7,25.1,32.2$, and 41.1 at. $\% \mathrm{Cr}$. The first four samples were annealed at $840^{\circ} \mathrm{C}$, and then cooled slowly. The last two were annealed at $1250^{\circ} \mathrm{C}$, and quenched in oil to avoid precipitation. Parker plots were constructed for all samples. Values of $\left(\Delta \rho / \rho_{0}\right)_{\mathrm{ph}}$ and $\left(\Delta \rho / \rho_{0}\right)_{\mathrm{im}}$ were found from 299 and $77 \mathrm{~K}$ data as before [Fig. 2(b)]. We also show the $\Delta \rho /$ $\rho_{0}=\left(\Delta \rho / \rho_{0}\right)_{\mathrm{ph}}$ value ${ }^{9}$ for pure $\mathrm{Fe}$ at room temperature. We see that $\left(\Delta \rho / \rho_{0}\right)_{\text {in }}$ may have a maximum at $x \simeq 5$ at. $\% \mathrm{Cr}$. Also, $\left(\Delta p / \rho_{0}\right)_{\mathrm{ph}}$ becomes negative as $x$ increases, and has a minimum at an $x$ value equal to or slightly larger than the one where $\left(\Delta \rho / \rho_{0}\right)_{\mathrm{im}}$ is maximum. This behavior is very similar to the one for $\mathrm{Ni}-\mathrm{Fe}$, and again cannot be explained by $D_{1}$ and $D_{1}$ variations.

Finally, we analyze existing data ${ }^{13}$ of $\Delta \rho / \rho_{0}$ and $\rho_{0}$ for $\mathrm{Fe}_{100-x} \mathrm{~V}_{x}$ alloys at 273,77 , and $4.2 \mathrm{~K}$ in the same way [Fig. 2(c) 1. Again, like in $\mathrm{Ni}-\mathrm{Fe}$ and $\mathrm{Fe}-\mathrm{Cr},\left(\Delta \rho / \rho_{0}\right)_{\mathrm{ph}}$ becomes negative at increasing $x$, and looks like an inverted mirror image of $\left(\Delta \rho / \rho_{0}\right)_{i m}$.

\section{SPLIT-BAND MODEL AND SATUPATION}

The existence of the $\left(\Delta \rho / \rho_{0}\right)_{\text {in }}$ maximum in $\mathrm{Ni}-\mathrm{Fe}$ has been attributed ${ }^{14}$ to a scattering resonance located in the spin-down $3 d$ band, at the boundary $T$ between the $N i$ and Fe subbands in the "split-band" model. A similar resonance exists ${ }^{14}$ in the spin-up $3 d$ band of $\mathrm{Fe}-\mathrm{Cr}, \mathrm{Fe}-\mathrm{V}$, etc. In $\mathrm{Ni}-\mathrm{Fe}$ alloys, point $T$ is predicted ${ }^{15}$ to pass through the Fermi level when $x=18$ at. $\%$ Fe. This interpretation is confirmed by an empirical data analysis ${ }^{i t}$ of $\Delta \rho / \rho_{0}$ data at $4.2 \mathrm{~K}$ performed by Szentirmay and Kedves, which is based on Eq. (1) and shows that $\rho_{1}$ has a large maximum $\approx 70 \times 10^{-8}$ $\Omega \mathrm{mat} \approx 15$ at. $\% \mathrm{Fe}$ while $\rho$, follows a smooth Nordheim behavior in that range.

Note that $\left(\Delta p / p_{0}\right)_{\text {im }}$ is relatively large in all these alloys, because ${ }^{14}$ of the resonance, and because they all follow the Slater-Pauling curve for magnetization.

Scattering resonances and split-band effects are one manifestation of the breakdown of the first Born approximation, happening ${ }^{14}$ because the valence difference $\mid Z$ | between solvent and solute in $\mathrm{Ni}-\mathrm{Fe}, \mathrm{Fe}-\mathrm{Cr}$, and $\mathrm{Fe}-\mathrm{V}$ reaches or ex- 


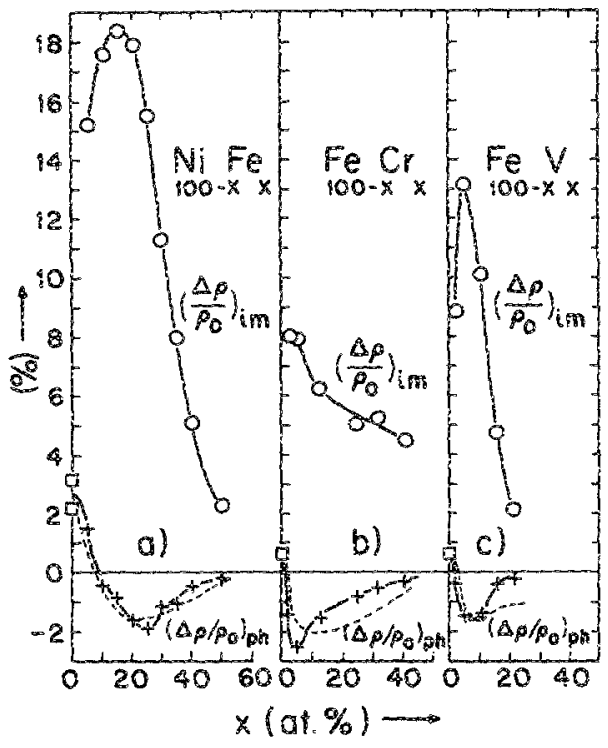

FIG. 2. (a) Circles represent $\left(\Delta \rho / \rho_{0}\right)_{\text {im }}$ value for $\Delta i_{100-x} \mathrm{Fe}_{x}$ as a function of $x_{3}$ while crosses represent $\left(\Delta \rho / \rho_{0}\right)_{p h}$. The squares are $\Delta p / \rho_{0}$ values for pure $N i$ at $\approx 290 \mathrm{~K}$. The dashed line is the prediction of $\mathrm{Eq}$. (4) for $\left(\Delta p / \rho_{0}\right)_{\text {plt }}$ (b) Same for $F e_{(02)}{ }_{x} \mathrm{Cr}_{x}$. The square is the $\Delta p / \rho_{0}$ value for pure $\mathrm{Fe}$ at roon temperature. (c) Same for $\mathrm{Fe}\left(100 \ldots \mathrm{V}_{x}\right.$.

ceeds 2. On the other hand, the Born approximation holds approximately in Fe-Co because $|Z|=1$.

It is well known ${ }^{17}$ that the temperature coefficient of the resistivity is smaller in alloys where the resistivity $\rho_{\mathrm{im}}$ caused by impurity scattering is large. We apply this idea of "resistivity saturation" to $\rho_{1}$ in alloys with a scattering resonance, and write

$$
\rho_{1}(T) \approx \rho_{1}^{\mathrm{im}}+\rho^{\mathrm{ph}}(T)-\left(\rho_{1}^{\mathrm{im}} / \rho_{\text {sat }}\right) \rho_{\mathrm{i}}^{\mathrm{ph}}(T)
$$

where $\rho_{!}^{\text {im }}$ and $\rho_{l}^{\text {ph }}(T)$ are the impurity and phonon resistivities in the absence of saturation, and $\rho_{\mathrm{sus}}>0$ is an adjustable parameter associated with saturation. By combining Eqs. (1) and (3) in the limit $\rho_{1} / \rho_{1} \geqslant 1$, we obtain

$$
\begin{aligned}
\left(\frac{\Delta \rho}{\rho_{0}}\right)_{p h}= & \left(\frac{\Delta \rho}{\rho_{0}}\right)_{p h}^{z}+\frac{D(x)}{D(0)}\left[1-\left(\frac{\Delta \rho}{\rho_{0}}\right)_{\mathrm{im}} \frac{\rho_{0}(4.2)}{\gamma \rho_{\mathrm{sat}}}\right] \\
& \times\left[\left(\frac{\Delta \rho}{\rho_{0}}\right)_{p h}(x=0)-\left(\frac{\Delta \rho}{\rho_{0}}\right)_{\mathrm{ph}}^{2}\right],
\end{aligned}
$$

where $\left(\Delta \rho / \rho_{0}\right)_{\mathrm{ph}}(x=0)$ is the value of $\left(\Delta \rho / \rho_{0}\right)_{\mathrm{ph}}$ for pure nickel. Also, the $\left(\Delta \rho / \rho_{0}\right)_{\text {oh }}^{z}$ terms are a correction representing the contribution " of the $L_{z} S_{z}$ part of the spin-orbit interaction to $\left(\Delta \rho / \rho_{0}\right)_{\mathrm{ph}}$, assumed indeperdent of $x$. Finally, $D(x) / D(0)$ is a correction factor taking into account the variation of the electronic density of states $D$ with $x$. Values of $D(x)$ are derived from electronic specific heat data of the literature. The equation should also hold for Fe-based alloys with a spin-up scattering resonance. The values of
$\left(\Delta \rho / \rho_{0}\right)_{\text {ph }}$ predicted by Eq. (4) are plotted as a dashed line on Fig. $2(\mathrm{a})$, assuming $\gamma \rho_{\text {sat }}=1.88 \times 10^{-8} \Omega \mathrm{m}$ and $\left(\Delta \rho / \rho_{0}\right)_{\mathrm{ph}}^{z}=-4 \%$, and using experimental values of $\left(\Delta \rho / \rho_{0}\right)_{\mathrm{im}}, \rho_{0}(4.2)$, and $\left(\Delta \rho^{\prime} \rho_{0}\right)_{\mathrm{ph}}(x=0)$. In the case of Figs. $2(\mathrm{~b})$ and $2(\mathrm{c})$, we use $\gamma p_{\mathrm{sat}}=1.25 \times 10^{-8} \Omega \mathrm{m}$, $\left(\Delta \rho / \rho_{0}\right)_{\mathrm{ph}}^{z}=-3 \%$. We see that the position and value of the minimum of $\left(\Delta \rho / \rho_{0}\right)_{p h}$ are predicted fairly well by Eq. (4), considering that Eo. (3) is a very rough approximation. This confirms the validity of our explanation of that minimum, in crystalline $\mathrm{Ni}-\mathrm{Fe}, \mathrm{Fe}-\mathrm{Cr}$, and $\mathrm{Fe}-\mathrm{V}$, based on the ideas of scattering resonance and resistivity saturation. Also, the $\gamma \rho_{\text {sai }}$ values above lead to $\rho_{\text {sat }} \simeq 125-188 \times 10^{-8} \Omega \mathrm{m}$, quite consistent with literature values. ${ }^{7}$

\section{ACKNOWLEOGENTS}

This work was supported in part by National Science Foundation Grant No. DMR 84-19980 and in part by the CMU Magnetics Technology Center. We are grateful to the University of Porto, Portugal, for allowing use of their equipment.

1. A. Campbell, A. Fert, and O. Jaow1, J. Phys. C (London) 3, $\$ 95$ (1970); A. Fert, X. Plyys C (London) 2, 1784 (1969).

${ }^{2}$ C. Herring, Phys. Rev. Let: 19, 167, (1967); 19, 684 (E) 1967; L. Colquitt, Phys. Rev. A 139, 1857 (1965).

${ }^{3} \mathrm{G}$. K. White, in Proceedings of the $84 \mathrm{~h}$ Conference on Thermal Conductiv ity, edited by C. Y. Hoand R. E. Taylor (Plenum, New York, 1969), p. 37 (see Fig. 3); G. K. White and S. B. Woods, Philos. Trans. R. Soc. London Ser. A. 251, 273 (1959) (see Table 5); Can. J. Phys. 35, 656 (1957) (see Fig. 4 ; ; actually, $L(T)$ seems to exceed $L_{\text {el }}$ somewhat at hight $T$, in the case of iron.

${ }^{4}$ R. Parker, Proc. Phys. Soc. London Sect. A 64, 447 (1951); \$ 64, 930 (1951); B 65, 616 (1952).

${ }^{5}$ L. Berger and D. Rivier, Heiv. Phys. Acka 35, 715 (1962) [see Eq. (6) and Fig. 101; L. Berger and S. A. Friedberg, Phys. Rev. 165, 670 (1968).

6P. P. Freitas and Z. Berger, J. Magn. Magn. Mater. 54-57, 1515 (1986). Fe-Co sample preperation is described in this paper.

72. Berger and A. R. de Vroomen, J. Appi. Plyss. 36, 277 7 (1965).

8. P. Freitas and L. Berger, Phys. Rev. B 37, 6079 (1988).

9J. Snit, Physica 16, 612 (1951).

${ }^{10}$ H. C. Yan Elst, Physica 25, 708 (1959); I. A. Campbell, J. Phys. F (London) 4, L181 (1974); R. M. Bozorth, Phys. Rev. 70, 923 (1946).

"Y. Shirakawa, Sci. Repts. Tohoku Imper. Univ. 27, 485 (1939); W. Bellinghats and M. P. de Andres, Ann. Ehys. 5, 187 (1960); T. Farrell and D. Greig, J. Phys. C (London) 1, 1359 (1968); M. C. Cadeville and B. Loegel, J. Phys. F (London) 3, L115 (1973); J. Smit, Physica 21, 877 (1955).

${ }^{2}$ E. Englert, Ann. Phys 589 (1932); T. R. McGuire, AlP Conf. Proc. 24,435 (1974).

${ }^{13}$ N. Sueda and H. Fujiwara, J. Sei. Hiroshima Univ. A 35, 59 (1971); N. Sueda, Y. Fujwara, and H. Fujiwara, ibid. 33, 267 (1969).

${ }^{14}$ L. Berger, AlP Conf. Proc. 34, 355 (1976).

1.5. Rerger, Physica 91B, 31 (1977).

${ }^{16} \mathrm{~J}$. Szentirmay and F. J. Kedves, Acta Universiratis Debreciensis, Ser. Phys. Chem., p. 21 (1978); also quoted on Fig. 5 of P. Muth and V. Christoph, $\bar{l}$. Phys. F (London) 11, 2119 (1981).

${ }^{17} \mathrm{Z}$. Fisk and O. W. Webb, Phys. Rev. Lett. 36,1084 (1976).

${ }^{18}$ O. Jaoul, 1. A. Campbell, and A. Fert, J. Magn. Magn. Mater. \$, 23 (1977). 